\title{
Acute renal insufficiency, secondary to the advanced pelvic organ prolapse and its resolution
}

\begin{abstract}
The hydronephrosis associated with advanced pelvic organ prolapse is known, in rare cases it causes severe acute or chronic renal failure. We present the case of a 73-yearold patient, who consulted for emergencies due to drowsiness and fatigue, symptoms associated with acute renal failure. At the physical examination of evidenced prolapse of pelvic organs (Grade IV), complementary tests report moderate bilateral hydronephrosis, acute renal failure and urinary sepsis. The diagnosis and reduction of urogenital prolapse through surgical treatment, demonstrates the resolution of the clinical picture, with adequate functional, aesthetic and social recovery in the patient.
\end{abstract}

Volume 3 Issue 2 - 2019

\author{
Imelda Magdalena Miranda Mazariegos \\ Unit of Gynecological Urology and Reconstructive Surgery of \\ the Pelvis, Former Civil Hospital of Guadalajara Fray Antonio \\ Alcalde, Mexico
}

Correspondence: Imelda Magdalena Miranda Mazariegos, Programa de Especialidad de Uroginecología y Reconstrucción de Piso Pélvico del Hospital Civil Fray Antonio Alcalde, Hospital No. 278, Colonia Centro, C.P 44280, Guadalajara, Jalisco, México, Email imeldamiranda20@hotmail.com

Received: August 05, 2018 | Published: March 25, 2019

\section{Introduction}

Pelvic organ prolapse (POP) is an important part of pelvic floor dysfunction, which may be associated with various symptoms of urinary dysfunction. Beyond POP, it is known that POP can be the cause of obstructive uropathy that can result in hydronephrosis. ${ }^{1,2}$ In the United States, a total of 200,000 surgeries are estimated for treatment of POP annually with an estimated risk of $12.6 \%$ of needing surgery at some point in life. ${ }^{3}$ This pathology is more prevalent among elderly, postmenopausal and multiparous women. One sixth of patients who have urogenital prolapse, essentially uterine prolapse, may have some degree of hydronephrosis. It has been documented that the prevalence of hydronephrosis in patients with pelvic organ prolapse varies between $7 \%$ and $17 \%, 4,5$ other studies have documented a prevalence of $22.5 \%{ }^{6}$ This is related and increases significantly with the severity of POP. The mechanism of hydronephrosis in patients with POP described by the literature suggests that hydronephrosis can result from the angulation of the ureters by extrinsic compression of the uterus in patients with advanced prolapse, while in patients with prolapse of the vaginal vault the cause of Hydronephrosis is due to the weakening and disintegration of the cardinal ligaments post hysterectomy. ${ }^{7-10}$ If hydronephrosis is not treated in a timely manner, it can progress to the development of acute renal failure and consequently to chronic renal failure. Hydronephrosis is the most serious complication of urogenital prolapse, it is usually corrected by surgical intervention of urogenital prolapse and reverts spontaneously in most cases.

\section{Case Report}

It is a female patient of 73 years of age, Mexican, widowed, with a pathological history of chronic hypertension under treatment. Without surgical history. Gynecoobstetric background of 4 gestas, 4 births. The patient is referred to the outpatient clinic of urogynecology at the Civil Hospital of Guadalajara, Jalisco, Mexico, for a sensation of heaviness and foreign body that protrudes at the vaginal level. At physical examination T/A $110 / 60 \mathrm{mmHg}$, heart rate of 71 beats/minute, temperature of $36.7^{\circ} \mathrm{C}$, gynecological examination shows a total pelvic organ prolapse (Grade IV). Initial laboratory studies are performed showing a blood count within normal parameters, electrolytes without alterations, urea nitrogen at $12 \mathrm{mg} / \mathrm{dl}$ and creatinine at $0.62 \mathrm{mg} / \mathrm{dl}$. Presurgical evaluation is requested according to hospital protocol, for surgical treatment. Two weeks later the patient goes to the hospital emergency, referring to nausea, fatigue and drowsiness. The patient is admitted, new laboratories are requested, hemogram showed 14,500 $\mathrm{mm} 3$, neutrophils $91.1 \%$, urinary sediment moderate leukocyturia was found, electrolytes with sodium alteration $149 \mathrm{meq} / 1$, potassium 5.9 meq/l, calcium $10.2 \mathrm{mg} / \mathrm{dl}$, urea nitrogen $17 \mathrm{mg} / \mathrm{dl}$, creatinine $1.7 \mathrm{mg} /$ dl. Renal ultrasound was performed, both kidneys were found with an adequate medullary cortical relationship, with moderate pyelocalic and ureteral ectasia, bilateral. It was assessed by the urogynecology and nephrology department that, faced with the suspicion of acute renal failure secondary to advanced pelvic organ prolapse, it was decided to perform surgery (Leford Colpocleisis) without complications. Twelve days after admission the patient was discharged, after the complete resolution of acute renal failure and POP. Subsequently, the clinical evolution and blood analysis values were satisfactory, with 3-month follow-up on the control renal ultrasound, the complete resolution of hydronephrosis.

\section{Discussion}

Advanced urogenital prolapse without medical and/or surgical treatment can cause an obstructive uropathy, this pathology being frequent in multiparous and postmenopausal women. ${ }^{2}$ Commonly, the diagnosis of obstructive uropathy (hydronephrosis, acute or chronic renal failure) is often delayed. ${ }^{4}$ Being one of the main and serious urological complications of advanced POP. In many publications it has been documented that urogenital prolapse can cause ureteral obstruction at the level of its lower third along with bladder and bowel function alterations. The possible mechanism of ureteral obstruction is the compression of these by the uterine vessels due to the stretching of the uterine ligament (cardinal ligament), the levator ani muscle or the fundus of the uterus; In many cases, this obstruction produces hydronephrosis, which is usually asymptomatic, but can lead to acute renal failure and eventually progress to chronic renal failure. ${ }^{8}$ Large cystoceles associated or not with uterine prolapse predispose to obstructive symptoms, the presence of residual urine in a chronic manner and rarely the presence of bilateral hydronephrosis with the possibility of producing impaired renal function. ${ }^{1-9}$ It has been observed that the prevalence of hydronephrosis in patients with uterine prolapse undergoing corrective surgery varies between $7 \%$ and $17 \%{ }^{4,5}$ Hydronephrosis is related to the degree of uterine prolapse and the presence of prolapse of the anterior compartment, which supports the theory that hydronephrosis is produced by a 
ureteral compression against the uterine fundus or by the stretching of the uterine ligaments. ${ }^{5}$ The main symptoms of urogenital prolapse are: discomfort at genital level, urinary and gastrointestinal tract that affect daily activities and quality of life of patients. ${ }^{3}$ In the case presented, it is concluded that advanced pelvic organ prolapse was the cause of hydronephrosis; Secondary to this the patient developed acute renal failure and urinary sepsis. We support our conclusion because after the surgical correction of the POP, the resolution of the clinical picture was evidenced, with an adequate functional, aesthetic and social recovery in the patient. Therefore, a suitable gynecological evaluation and renal ultrasound is suggested as part of the initial cabinet exams in patients with advanced POP, since it would be useful in the detection and prioritization of patients requiring immediate surgical treatment.

\section{Acknowledgments}

None.

\section{Conflicts of interest}

The author declares there is no conflicts of interest.

\section{References}

1. Beverly CM, Walters MD, Weber AM. Prevalence of hydronephrosis in patients undergoing surgery for pelvic organ prolapse; Obstet Gynecol vaginal vault prolapse). Inc. Kimberly Kenton editor. 2017.

2. Mark D Walters, Mickey M Karram. Uroginecologia y Cirugía Reconstructiva de la pelvis. Elsevier Masson, 3rd edn. 2014. p. 43-52.
3. AM Weber, P Abrams, L Brubaker, et al. The Standardization of Terminology for Researchers in Female Pelvic Floor Disorders. International Urogynecology Journal. 2001;12(3):178-186.

4. Ofer Gemer, Marina Bergman, Shmuel Segal. Prevalence of hydronephrosis in patients with genital prolapse. European Journal of Obstetrics \& Gynecology and reproductive biology. 1999;86(1):11-13.

5. Beverly CM, Walters MD, Weber AM, et al. Prevalence of hydronephrosis in patients undergoing surgery for pelvic organ prolapse. Obstet Gynecol. 1997;90(1):37-41.

6. Miranda-Mazariegos IM, Velázquez Castellanos PI Godoy-Rodríguez N, Orozco-Castellanos RJ. Prevalencia de hidronefrosis en mujeres con prolapso de órganos pélvicos avanzado. Rev Mex Urol. 2018;78(3)197201

7. Moore S, Bailey RR, Maling TM. Urinary tract obstruction and renal failure due to uterine prolapse. $N Z$ Med J. 1978;87:429-431.

8. Gemer O, Bergman M, Segal S. Prevalence of hydronephrosis in patients with genital prolapse. Eur J Obstet Gynecol Reprod Biol. 1999;86(1):11-19

9. Wein, Kavoussi, Novick. Peters; Obstrucción y traumatismo del tracto urinario superior; Campbell- Walsh Urology 10ma. 2015;41;11401186.

10. Mark D Walters, Mickey M Karram. Uroginecologia y Cirugía Reconstructiva de la pelvis. Elsevier Masson, 3rd edn. 2014;4:43-52. 